\title{
Optimization of Simple Sphygmomanometric Blood Pressure Measurement in Routine Prenatal Care
}

\author{
Vonck $\mathrm{S}^{1,2 *}$, Oben $\mathrm{J}^{2}$, Staelens $\mathrm{AS}^{2}$, Lanssens $\mathrm{D}^{1,2}$, Molenberghs $\mathrm{G}^{4}$ and Gyselaers $\mathbf{W}^{1-3}$ \\ ${ }^{1}$ University of Hasselt, Faculty of Medicine and Life Sciences, Belgium \\ ${ }^{2}$ Department of Obstetrics and Gynecology, Ziekenhuis Oost-Limburg, Genk, Belgium \\ ${ }^{3}$ Department Physiologies, Hasselt University, Hasselt, Belgium \\ ${ }^{4}$ Center for Medical Statistics, Hasselt University, Belgium
}

\begin{abstract}
Background: Despite reported early subclinical hypertension of women at risk, blood pressures at threshold 140/90 $\mathrm{mm} \mathrm{Hg}$ are used today to guide prenatal care. We aim to investigate the most appropriate gestation-specific threshold to measure early gestational blood pressures, allowing for a simple stratification between pregnant women at low/high risk for hypertension.

Methods: Singleton pregnancies were selected at Clinic Oost-Limburg, Genk, Belgium. A standard protocol was used to measure systolic (SBP), diastolic (DBP) and mean arterial pressure (MAP) in supine and standing position, by mode of an oscillometric sphygmomanometer around 12 and 20 weeks of gestation. After delivery, outcome was categorized in normotensive or hypertensive pregnancies. In a subgroup, routine blood pressures retrieved from prenatal records were compared to standardized blood pressures. ROC analysis was used to define early gestational blood pressure thresholds with best discriminative performance for hypertension. All analyses were done in SPSS software $(\alpha \leq 0.05)$.

Results: A total of 780 women were measured at 12 weeks, of which 433 pregnant women were re-evaluated around 20 weeks. At both occasions, blood pressures were significantly higher in hypertensive than in normotensive pregnancies $(p<0.0001)$. Analysis showed for DBP in standing position at cut off $79 \mathrm{mmHg}$ a sensitivity, specificity, positive predictive value and negative predictive value of $72 \%, 64 \%, 15,5 \%$ and $96 \%$ at 12 weeks and $86 \%, 69 \%, 20 \%$ and $98 \%$ at 20 weeks at cut off $77 \mathrm{~mm} \mathrm{Hg}$. At 20 weeks, Area under the Curve (AUC) for DBP was $83 \%$ in standing position and $80 \%$ in supine position. For routine versus standardized blood pressure measurement, AUC was $66 \%$ versus $72 \%$ at 12 weeks and $69 \%$ versus $82 \%$ at 20 weeks, respectively.
\end{abstract}

Conclusion: Simple blood pressure measurements with gestation-specific thresholds can easily be used worldwide towards improved planning of prenatal care as compared to current protocols.

Keywords: Blood pressures; Sphygmomanometer; Threshold; Hypertension; Pregnancy; Screening

\section{Introduction}

During normal pregnancy, the blood pressure decreases in the first 20 weeks and then gradually returns to preconceptional values when term approaches $[1,2]$. Around $6-8 \%$ of all expectant mothers will experience an aberrant blood pressure pattern leading to diverse hypertensive disorders, with maternal and neonatal mortality and morbidity such as cardiac arrest, renal failure, premature delivery, low birth weight, intra-uterine growth restrictions etc. [3,4]. Usually their blood pressure will remain stable during the first 20 weeks, where after an increase will initiate noticed in third trimester [5].

Today, a minimum of $140 / 90 \mathrm{~mm} \mathrm{Hg}$ measured at 2 occasions, $>6 \mathrm{~h}$ apart, is generally used as threshold to diagnose hypertension in pregnancy [6]. Hypertension already noticed at $<20$ weeks of gestation is labelled essential or chronic hypertension, and is considered a major risk factor for preeclampsia [7]. However, in most cases of gestational hypertension and preeclampsia, the hypertension is diagnosed at $\geq$ 20 weeks of gestation in women with "normal" blood pressure values earlier in pregnancy [6].

It is known that maternal cardiovascular (mal) adaptation during the first weeks of gestation plays a fundamental role in the regulation of blood pressure and the development of later hypertensive disorders $[8,9]$. Several studies $[1,10,11]$ report already higher blood pressure values around 12 weeks of gestation in women who eventually develop preeclampsia, gestational or essential hypertension, compared to women who remain normotensive. Today, subclinical higher blood pressures $(<140 / 90 \mathrm{~mm} \mathrm{Hg})$ are not considered clinically relevant. In this study, we evaluated whether early gestational diagnosis of subclinical hypertension would be helpful to identify the proportion of women destined to develop GHD, as early identification of those high risk cases could help decreasing the maternal and neonatal consequences [12].

\section{Methods}

\section{Patients}

Women with singleton pregnancies in first or second trimester, attending the obstetric ultrasound clinic (Ziekenhuis Oost-Limburg, Genk, Belgium) for their routine obstetrics scans, were invited to

*Corresponding author: Sharona Vonck, Department of Obstetrics and Gynecology, University of Hasselt, Ziekenhuis Oost-Limburg, Schiepse Bos 6 3600 Genk, Belgium, Tel: +32 8932 15 15; E-mail: sharona.vonck@zol.be

Received January 06, 2017; Accepted February 06, 2017; Published February 13, 2017

Citation: Vonck S, Oben J, Staelens AS, Lanssens D, Molenberghs G, et al. (2017) Optimization of Simple Sphygmomanometric Blood Pressure Measurement in Routine Prenatal Care. Health Care Current Reviews 5: 185. doi: 10.4172/2375 4273.1000185

Copyright: $\odot 2017$ Vonck S, et al. This is an open-access article distributed unde the terms of the Creative Commons Attribution License, which permits unrestricted use, distribution, and reproduction in any medium, provided the original author and source are credited. 
participate in a prospective, observational study. Approval of the Ethical Committee was obtained before study onset (MEC ZOL, reference: 13/090U). Blood pressures were measured as part of a reported standardized non-invasive cardiovascular assessment protocol using impedance cardiography (ICG), combined ECG/Doppler sonography and bio-impedance [13-15]. Oral informed consent was obtained before inclusion. At birth, gestational outcome was defined and categorized in normotensive pregnancy (NP) or hypertensive pregnancies (HP), which included gestational hypertension $(\mathrm{GH})$, preeclampsia (PE) and essential hypertension (EH). GH was diagnosed when a high blood pressure ( $\geq 140 / 90 \mathrm{~mm} \mathrm{Hg}$ ) was observed after midpregnancy, without proteinuria, twice measured with $6 \mathrm{~h}$ in between. The diagnosis of $\mathrm{PE}$ was determined when gestational hypertension was accompanied with de novo proteinuria ( $\geq 300 \mathrm{mg}$ per $24 \mathrm{~h}$ ). EH was defined as hypertension present before pregnancy or before 20 weeks of gestation [6]. Pregnancies diagnosed with isolated intra-uterine growth retardations (IUGR) and/or multiples were excluded from analysis as they are reported with different cardiovascular profiles $[16,17]$. Additionally, demographic details were recorded: maternal age (years), pregestational BMI, gestational age at assessment and at delivery, parity, cigarette usage, medication, neonatal birth weight and percentile.

\section{Protocol}

The systolic blood pressure (SBP), diastolic blood pressure (DBP) and mean arterial pressure (MAP) of all patients were measured and registered around 12 weeks and/or 20 weeks of gestation. The blood pressure measurements were performed as part of the Non-Invasive Continuous Cardiac Output Monitor assessment (NICCOMO, Medis Medizinische Messtechnik GmbH, Ilmenau, Germany) by use of an oscillometric sphygmomanometer at standardized time-points in a previously reported protocol [18]. Each patient performed the complete ICG examination first in supine position and afterwards in standing position. Before the first blood pressure measurement, the patient was already comfortably in supine position for $5 \mathrm{~min}$. 1,5 min after supine blood pressure measurement, the patient changed position to standing. The second blood pressure measurement was taken after a rest period of $2 \mathrm{~min}$ in standing position (Figure 1). Blood pressures were always taken on the right arm and with an appropriate cuff width.

\section{Statistics}

Normality was checked via Shapiro-Wilk for continuous variables. Even though there is a comparison of several parameters at two different time points (12 weeks and 20 weeks), each comparison is of the two-group type. To this end, the non-parametric Mann Whitney $U$ was used at $a<0,05$ to test the null hypothesis whether the distribution of the parameter of interest, at a given time point, is identical between the two groups. For categorical variables, Chi-square test was done. Data are presented as median and interquartile ranges (IQR) or $n$ (\%). ROC analysis was used to examine the different thresholds for each blood pressure in standing and supine position and sensitivity, specificity, positive and negative predictive value for prediction of

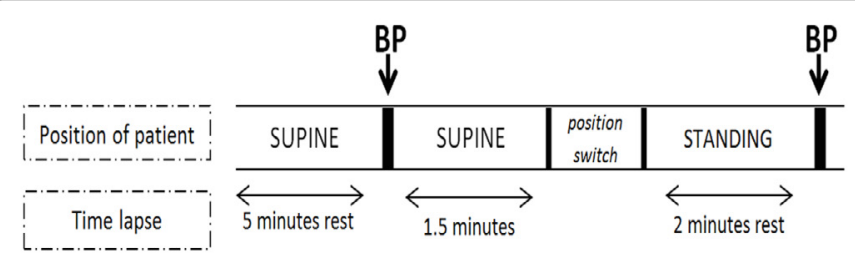

Figure 1: Graphical representation of the protocol. hypertension were calculated. Youdens Index was used to identify the most appropriate threshold for every blood pressure measurement. All analyses were done in SPSS (SPSS Inc., Chicago, Illinois, USA).

\section{Sub analysis}

In a subgroup of the studied population, the standardized blood pressure values, measured as explained above, were compared to the blood pressures values at corresponding gestational age recorded in the prenatal files as part of the routine prenatal visits by the obstetrician or midwife. These latter blood pressures values were retrieved retrospectively from the patient's records. Paired t-tests at nominal level $\alpha=0.05$ and Pearson Correlations Coefficients (PCC) were calculated between blood pressures measured in the standardized conditions versus the blood pressures measured at prenatal visit. All analyses were done in SPSS (SPSS Inc., Chicago, Illinois, USA).

\section{Results}

A total of 870 pregnant women had first trimester standardized blood pressure measurements; 433 of those women also had second trimester measurements. After birth, 716 patients were classified as normotensive and 64 as hypertensive patients at 12 weeks. At 20 weeks, 398 normotensive patients and 35 hypertensive patients were measured. A total of 90 pregnancies with isolated IUGR were excluded. The hypertensive group in first trimester included 24 women with GH (37.5\%), 22 (34.5\%) with PE and 18 (28\%) with EH. A summary on maternal demographics is shown in Table 1. In the HP group, compared with NP, women were heavier, older and the nulliparity percentage is higher. The general use of medication is also significantly higher in the HP group, where $65 \%$ consists of blood pressure medication vs. $1.5 \%$ blood pressure medication in the NP group. Hypertensive patients delivered at an earlier gestational age and their neonates had a lower birth weight Due to the slight differences between maternal age, pre-pregnancy BMI and nulliparity between HP and NP (Table 1), the comparisons done were supplemented with linear regression analyses, comparing groups while correcting for potential confounders. The results are identical in the sense that all group comparisons remain highly significant.

The 12 and 20 week blood pressures (SBP, DBP and MAP) of NP and HP were compared and all values were significantly higher in HP at both gestational ages (Table 2). There were conducted several comparisons here and it is therefore prudent to apply a multiple comparisons correction. However, given the highly significant nature of the test statistics and the relatively modest number of tests, an adjusted alpha level still leaves the results highly significant. Indeed, for the 12 tests reported in Table 2, the adjusted alpha level would be 0.00417; all p-values reported are well below this threshold.

ROC analysis was used to evaluate the performance of SBP, DBP or MAP in supine and standing position to predict the hypertensive cases. The most appropriate thresholds for each blood pressure were identified via the Youdens Index (Table 3). Based on the AUC and Youdens Index, the DBP in standing position around 12 weeks and 20 weeks of gestation showed the best performance (Figure 2). This represents for 12 weeks at cut off $79 \mathrm{mmHg}$ a $72 \%$ sensitivity, $64 \%$ specificity, $15,5 \%$ positive predictive value and $96 \%$ negative predictive value. Similarly at 20 weeks, a cut off of $77 \mathrm{mmHg}$ showed an $86 \%$ sensitivity, $69 \%$ specificity, $20 \%$ positive predictive and $98 \%$ negative predictive value. Despite a significant difference between standing and supine blood pressure $(p<0.004)$, the AUC's between standing and supine do not vary so much. At 20 weeks, AUC for DBP was $83 \%$ in standing position and $80 \%$ in supine position. 
Citation: Vonck S, Oben J, Staelens AS, Lanssens D, Molenberghs G, et al. (2017) Optimization of Simple Sphygmomanometric Blood Pressure Measurement in Routine Prenatal Care. Health Care Current Reviews 5: 185. doi: 10.4172/2375-4273.1000185

Page 3 of 6

\begin{tabular}{|c|c|c|c|}
\hline & NP & HP & p-value \\
\hline & $(n=716)$ & $(n=64)$ & \\
\hline \multicolumn{4}{|l|}{ Characteristics at inclusion } \\
\hline Maternal age, years & $30(27,33)$ & $31(28,35)$ & 0.029 \\
\hline Gestational age at inclusion, weeks & $\begin{array}{c}12 \text { w } 2 d \\
(11 w 5 d, 12 \text { w } 5 d)\end{array}$ & $\begin{array}{c}12 \text { w } 2 d \\
(11 w 4 d, 12 w 5 d)\end{array}$ & 0.557 \\
\hline Pre-pregnancy BMI, kg/m² & $23(21,26)$ & $24(22,28)$ & 0.028 \\
\hline Nulliparity & $341(47.6 \%)$ & $40(62.5 \%)$ & 0.023 \\
\hline Cigarette smoker & $67(9.4 \%)$ & $4(6.3 \%)$ & 0.408 \\
\hline Chronic Hypertension & 0 & $18(28 \%)$ & -- \\
\hline Gestational Hypertension & 0 & $24(37.5 \%)$ & -- \\
\hline Preeclampsia & 0 & $22(34.5 \%)$ & -- \\
\hline Medication & $60(8.4 \%)$ & $20(31.3 \%)$ & 0.0001 \\
\hline \multicolumn{4}{|l|}{ Outcome characteristics } \\
\hline Birth weight, g & $3425(3145,3750)$ & $3205(2740,3670)$ & 0.001 \\
\hline Birth weight, percentile & $57(35,77)$ & $52(27,77)$ & 0.39 \\
\hline Gestational age at delivery, weeks & $\begin{array}{c}39 w 5 d \\
(38 w 5 d, 40 w 4 d)\end{array}$ & $\begin{array}{c}38 w 5 d \\
(36 w 6 d, 39 w 6 d)\end{array}$ & 0.0001 \\
\hline
\end{tabular}

Data are presented as medians with interquartile ranges or $\mathrm{n}(\%)$. Differences between NP and HP are presented as $p$-values $\alpha<0,05$ was considered significant

Table 1: Patient and outcome characteristics of normotensive pregnancies (NP) and hypertensive pregnancies (HP).

\begin{tabular}{|l|c|c|c|}
\hline & \multicolumn{2}{|c|}{12 w } & \multicolumn{2}{|c|}{ NP } \\
\hline & NP & HP & \\
\hline SBP standing $(\mathrm{mm} \mathrm{Hg})$ & $115(108-124)$ & $124(116-134)$ & $113(105-121)$ \\
\hline DBP standing $(\mathrm{mm} \mathrm{Hg})$ & $75(71-81)$ & $82.5(78-90)$ & $73(68-78)$ \\
\hline MAP standing $(\mathrm{mm} \mathrm{Hg})$ & $85(81-91)$ & $92(88-102)$ & $83(78-88)$ \\
\hline SBP supine $(\mathrm{mm} \mathrm{Hg})$ & $113(106-121)$ & $123.5(116-141)$ & $110(103-119)$ \\
\hline DBP supine $(\mathrm{mm} \mathrm{Hg})$ & $70(66-75)$ & $75(71-85)$ & $68(78-89)$ \\
\hline MAP supine $(\mathrm{mm} \mathrm{Hg})$ & $80(75-85)$ & $86.5(82-96)$ & $122(114-133)$ \\
\hline
\end{tabular}

Data are presented as medians with interquartile ranges. All comparisons were done using Mann Whitney $U$ test

P-values are $<0,0001$

Table 2: Median+IQR for standardized SBP, DBP and MAP in supine and standing position at 12 weeks and 20 weeks.

IQR: Interquartile Range; SBP: Systolic Blood Pressure; DBP: Diastolic Blood Pressure; MAP: Mean Arterial Pressure; NP: Normotensive Pregnancies; HP: Hypertensive Pregnancies.

\begin{tabular}{|c|c|c|c|c|c|}
\hline & AUC $(95 \% \mathrm{Cl})$ & Threshold & Sensitivity & Specificity & FPR \\
\hline \multicolumn{6}{|c|}{12 WEEKS } \\
\hline SBP standing & $0.696(0.631-0.762)$ & $122 \mathrm{~mm} \mathrm{Hg}$ & 0.61 & 0.69 & 0.31 \\
\hline DBP standing & $0.744(0.68-0.808)$ & 79 mm Hg & 0.72 & 0.64 & 0.36 \\
\hline MAP standing & $0.744(0.683-0.805)$ & 87 mm Hg & 0.81 & 0.56 & 0.44 \\
\hline SBP supine & $0.726(0.659-0.793)$ & $116 \mathrm{~mm} \mathrm{Hg}$ & 0.76 & 0.60 & 0.40 \\
\hline DBP supine & $0.739(0.678-0.799)$ & $71 \mathrm{~mm} \mathrm{Hg}$ & 0.81 & 0.54 & 0.46 \\
\hline MAP supine & $0.744(0.681-0.806)$ & 83 mm Hg & 0.73 & 0.64 & 0.36 \\
\hline \multicolumn{6}{|c|}{20 WEEKS } \\
\hline SBP standing & $0.712(0.624-0.799)$ & $111 \mathrm{~mm} \mathrm{Hg}$ & 0.89 & 0.44 & 0.56 \\
\hline$D B P$ standing & $0.829(0.765-0.893)$ & 77 mm Hg & 0.86 & 0.69 & 0.31 \\
\hline MAP standing & $0.805(0.732-0.877)$ & 86 mm Hg & 0.89 & 0.61 & 0.39 \\
\hline SBP supine & $0.731(0.644-0.819)$ & $118 \mathrm{~mm} \mathrm{Hg}$ & 0.69 & 0.71 & 0.29 \\
\hline$D B P$ supine & $0.798(0.724-0.871)$ & $71 \mathrm{~mm} \mathrm{Hg}$ & 0.80 & 0.63 & 0.37 \\
\hline MAP supine & $0.810(0.739-0.880)$ & $81 \mathrm{~mm} \mathrm{Hg}$ & 0.83 & 0.66 & 0.34 \\
\hline
\end{tabular}

Table 3: Detection performance of blood pressures. Sensitivity, specificity and FPR of each blood pressure, when using the most appropriate threshold indicated by ROC analysis and Youdens Index at 12 weeks and 20 weeks.

AUC: Area under the Curve; FPR: False-Positive Rate; SBP: Systolic Blood Pressure; DBP: Diastolic Blood Pressure; MAP: Mean Arterial Pressure

The sub analysis in $262(33.6 \%)$ first trimester and $141(32.6 \%)$ second trimester pregnancies showed a significant, but weak correlation between standardized DBP (stDBP) and routine DBP (rDBP) $(12 \mathrm{w}$ :
PCC $=0.425, \mathrm{p}<0.0001 ; 20 \mathrm{w}: \mathrm{PCC}=0.429, \mathrm{p}<0.0001)$. Paired t-test comparison revealed significant differences between stDBP and $\mathrm{rDBP}$ at 12 weeks (76 $\pm 7 \mathrm{~mm} \mathrm{Hg}$ vs. $71 \pm 9 \mathrm{~mm} \mathrm{Hg}$ resp., $\mathrm{p}<0.0001)$ and 20 

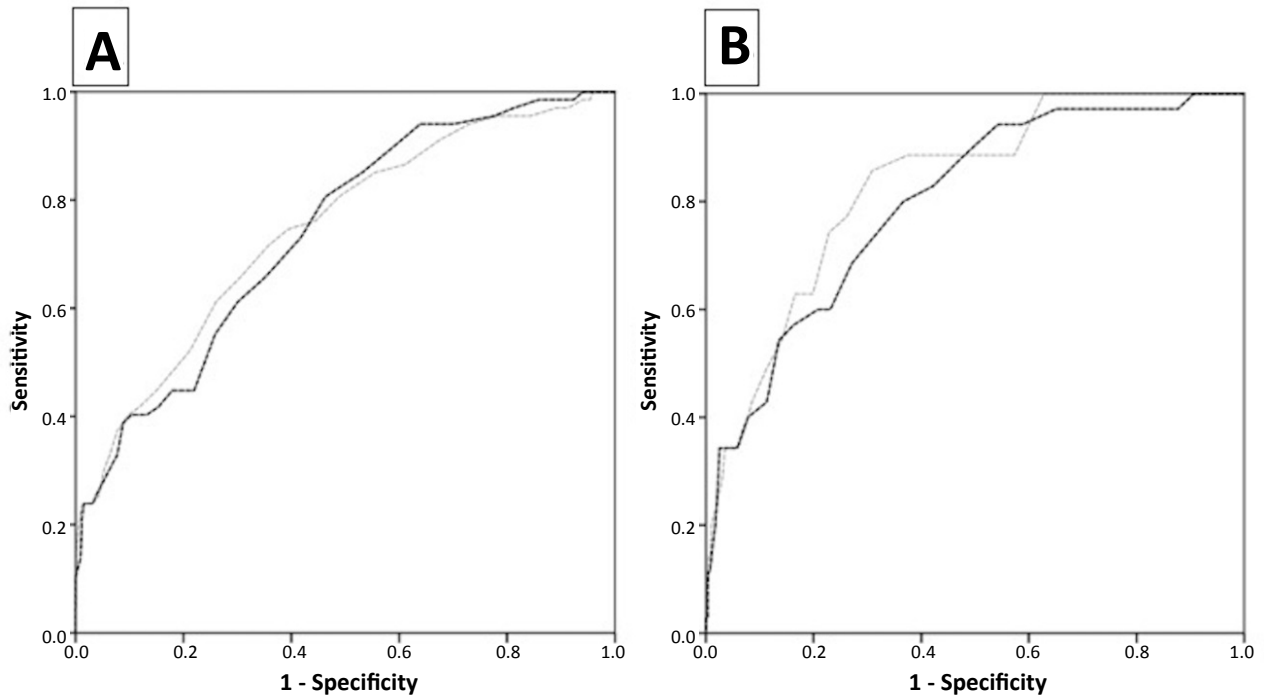

Figure 2: A: DBP ROC curves at 12 weeks, where grey indicates DBP standing (AUC: 0.744 ) and black indicates DBP supine (AUC: 0.739 ) B: DBP ROC curves at 20 weeks, where grey indicates DBP standing (AUC: 0.829) and black indicates DBP supine (AUC: 0.798). DBP: Diastolic Blood Pressure; ROC: Receiver Operating Characteristic; AUC: Area Under the Curve

weeks $(73 \pm 7 \mathrm{mmHg}$ vs. $69 \pm 8 \mathrm{~mm} \mathrm{Hg}$ resp., $\mathrm{p}<0.0001)$ of gestation. AUC of routine versus standardized BP measurement was $66 \%$ versus $72 \%$ at 12 weeks and $69 \%$ versus $82 \%$ at 20 weeks, respectively.

\section{Discussion}

This study illustrates the potential of simple sphygmomanometric blood pressure measurements in early pregnancy for diagnosing subclinical hypertension. In our study, a standardized measurement of DBP in standing position showed the best performance with threshold $79 \mathrm{~mm} \mathrm{Hg}$ at 12 weeks and $77 \mathrm{~mm} \mathrm{Hg}$ at 20 weeks. These observations suggest that the general threshold of $140 / 90 \mathrm{~mm} \mathrm{Hg}$ should be adjusted in function of the gestational age, as the use of a lower threshold improves the early predictability of hypertension in pregnancy. Our findings are relevant to all prenatal care workers because (rudimentary) early gestational screening for hypertensive disease becomes universally available worldwide using a very simple and already generally applied technique of sphygmomanometric blood pressure measurement, without the need for other technologies or expensive devices.

The strength of this study is the rigid standardized protocol for measuring the blood pressures and the amount of inclusions. All women gave birth in the same hospital, where data on gestational, maternal and neonatal outcome were traceable in the hospital records. Our study population is however not yet large enough to adjust the general used threshold of 140/90 mm Hg in clinic. The FPR presented in this paper can be lowered with implementation of other clinical or physical parameters to the screening process, but the lower thresholds can already serve as first discriminant tool seen the NPV is $96 \%$ at 12 weeks. With this accuracy, the focus lies not completely on detecting the hypertensive cases (as PPV is $15-20 \%$ ), but more on eliminating the healthy cases with more certainty.

Subclinical higher blood pressures $(<140 / 90 \mathrm{~mm} \mathrm{Hg})$ in the first half of pregnancy are reported as a first sign of hypertension $[1,19]$. Our data are in line with this. Hypertensive disorders during pregnancy result from maternal cardiovascular maladaptation, initiated during the first weeks of gestation. Normally a physiological cascade is initiated by the fall of the peripheral vascular resistance, whereby heart rate and cardiac output increase. This vasodilatation lowers the blood pressures, because the cardiac output incline is not sufficient to prevent a blood pressure fall. Systolic as well as diastolic blood pressure keeps decreasing until 24 weeks. The peripheral vascular resistance is shown to be higher in future hypertensive patients and do not experience a blood pressure fall [20].

Based on our results, a DBP above $79 \mathrm{~mm} \mathrm{Hg}$ at 12 weeks or above $77 \mathrm{~mm} \mathrm{Hg}$ at 20 weeks identifies 2-3 times more patients at risk than the currently used threshold of $90 \mathrm{~mm} \mathrm{Hg}$ in the first and second trimester. We have at 12 weeks a $72 \%$ sensitivity and $64 \%$ specificity, but this improves when measuring at 20 weeks again: $86 \%$ sensitivity and $69 \%$ specificity. This suggests that the current obstetric practice may benefit from changing the currently used 'gold standard of blood pressure measurement' by using different and gestation specific cut off values, a suggestion which has already be postulated by Hermida et al. [21]. This may be useful to all clinics where technologies to screen for gestational hypertensive disease are not available and potentially to those women considered for initiating preventive medications such as low-dose aspirin [22,23] or calcium [24]. Another opportunity could be the implementation of home blood pressure monitoring devices as follow up method, which - when used under standardized conditions- may offer valuable information without the need for increasing the number of prenatal visits or the risk of unnecessary medication intervention $[25,26]$. One study by Penny et al. on ambulatory automated blood pressure monitoring showed that a cut off of $135 / 85 \mathrm{~mm} \mathrm{Hg}$ had a better positive predictive value than 140/90 mm Hg [27]. Also Gallery et al. identified lower 'at risk' blood pressure values at 17-20 weeks: the risks for hypertension were higher with blood pressure values above $110 / 75 \mathrm{~mm} \mathrm{Hg}$ sitting or 100/65 mm Hg lying in left lateral position [1]. The latter group also observed that the fall in systolic and diastolic blood pressure from preconception to midgestation was larger in the normotensive than the hypertensive group, which is completely in line with the physiology or mentioned above [1].

Our data also emphasizes the relevance of posture: standing blood pressure values were significantly higher than the supine values. The heart is required to pump more blood to the brain and needs therefore 
a higher pressure. As mentioned above in the study of Gallery, cut off values depend on how the patients are positioned and thus it is important to interpret the observations relative to position specific thresholds too [1]. Applied on our study, based on AUC's, we noticed a slightly better predictive outcome with standing blood pressures instead of supine blood pressures. There is however some inconsistency concerning the influence of position on the blood pressure: lower supine blood pressures are reported as compared to sitting [1,28,29], but also higher values have been observed [30] as well as no differences at all $[31,32]$. These conflicting results may relate to different populations or other methods of measurement.

This paper emphasizes the importance of a standard protocol, as the reliability of blood pressure measurements in routine care seems rather low [33]. Values obtained under standardized, calm conditions in a consequent position are more informative than those obtained as a routine clinical activity. Already in the 90s, some authors discussed that identification of high risk patients is better with an automated blood pressure protocol instead of a conventional measurement in the antenatal clinic. The clinical readings are influenced by inaccuracy due to observer bias, presence of the doctor (white coat hypertension), device bias etc. $[27,34,35]$. Benedetto et al. promotes a $24 \mathrm{~h}$ blood pressure monitoring to estimate the risk. Aside the fact that Benedetto et al. also suggests lower blood pressure thresholds based on his results, it is easier to perform only a short protocol on each patient in clinic instead of a $24 \mathrm{~h}$ protocol [19]. Since blood pressure values are the main indicator of hypertension, doctors and midwives should pay more attention to the measurement technique and to opting for the best performing protocol which is gestation-specific, position-specific, populationspecific and possibly clinic-specific depending on the used method [1,36-38]. Reports mention that $45 \%$ of the obstetricians never use an appropriate cuff [39] or $66 \%$ report the blood pressure to the nearest 5 $\mathrm{mm} \mathrm{Hg}$, which automatically leads to over or under estimation [40]. Routine antenatal visits are troubled with fast and non-standardized blood pressure measurement in patients who had to sit in the waiting room for minutes to hours or were rushed because of an appointment delay $[37,38]$. This scenario is to be prevented with a standardized protocol, where the measurement of taking the blood pressure is a real moment. Ciccone et al. showed improved clinical outcome of patients suffering from cardiovascular diseases, diabetes or heart failure due to the active clinical implementation of health care managers (specially trained nurses) as compared to a doctor visit alone [41]. Parallel to this study, midwives could be an added value in the disease management for gestational hypertensive disorders.

From the data presented in this paper, we conclude that a simple blood pressure measurement, if measured in a standardized way, can already be very valuable to classify the complete patient population into high vs. low risk at their first prenatal visit. This might be an important starting point for a universal screening tool, certainly for patients in their first pregnancy. Applying a threshold of $79 \mathrm{~mm} \mathrm{Hg}$ at 12 weeks or $76 \mathrm{~mm} \mathrm{Hg}$ at 20 weeks gives us at least a $\geq 96 \%$ negative predictive value, which is helpful to exclude hypertensive disorders early in pregnancy, leaving a "high" risk group requiring a somewhat closer observation or advanced screenings tests with multi-marker algorithms.

\section{Acknowledgement}

The first author of this work is funded by a Ph.D. grant of the Agency for Innovation by Science and Technology (IWT) in Brussels, Belgium and all authors are part of the Limburg Clinical Research Project (LCRP) at Hasselt University, Belgium. We also want to thank Dr. Petra Nijst for her input on the paper.

\section{References}

1. Gallery ED, Hunyor SN, Ross M, Gyory AZ (1977) Predicting the development of pregnancy-associated hypertension. The place of standardised bloodpressure measurement. Lancet 1: 1273-1275.

2. Reiss RE, O'Shaughnessy RW, Quilligan TJ, Zuspan FP (1987) Retrospective comparison of blood pressure course during preeclamptic and matched control pregnancies. Am J Obstet Gynecol 156: 894-898.

3. Uzan J, Carbonnel M, Piconne O, Asmar R, Ayoubi JM (2011) Pre-eclampsia Pathophysiology, diagnosis, and management. Vasc Health Risk Manag 7 : 467-474.

4. Duley $L$ (2009) The global impact of pre-eclampsia and eclampsia. Semin Perinatol 33: 130-137.

5. Ayala DE, Hermida RC, Mojon A, Fernandez JR, Silva I, et al. (1997) Blood pressure variability during gestation in healthy and complicated pregnancies. Hypertension 30: 611-618.

6. Report of the National High Blood Pressure Education Program Working Group on High Blood Pressure in Pregnancy. Am J Obstet Gynecol 183: S1-1S22.

7. Rey E, Couturier A (1994) The prognosis of pregnancy in women with chronic hypertension. Am J Obstet Gynecol 171: 410-416.

8. Steegers EA, von Dadelszen P, Duvekot JJ, Pijnenborg R (2010) Preeclampsia. Lancet 376: 631-644.

9. Sep SJ, Schreurs MP, Bekkers SC, Kruse AJ, Smits LJ, et al. (2011) Early pregnancy changes in cardiac diastolic function in women with recurrent preeclampsia and in previously pre-eclamptic women without recurrent disease. BJOG: An International Journal of Obstetrics and Gynaecology 118: 1112-1119.

10. Iwasaki R, Ohkuchi A, Furuta I, Ojima T, Matsubara S, et al. (2002) Relationship between blood pressure level in early pregnancy and subsequent changes in blood pressure during pregnancy. Acta Obstet Gynecol Scand 81: 918-925.

11. Oben J, Tomsin K, Mesens T, Staelens A, Molenberghs G, et al. (2014) Maternal cardiovascular profiling in the first trimester of pregnancies complicated with gestation-induced hypertension or fetal growth retardation: A pilot study. $J$ Matern Fetal Neonatal Med 27: 1646-1651.

12. National High Blood Pressure Education Program Working Group Report on High Blood Pressure in Pregnancy. Am J Obstet Gynecol 163: 1691-1712.

13. Tomsin K, Mesens T, Molenberghs G, Gyselaers W (2012) Impedance cardiography in uncomplicated pregnancy and pre-eclampsia: A reliability study. J Obstet Gynaecol 32: 630-634.

14. Gyselaers W, Molenberghs G, Van Mieghem W, Ombelet W (2009) Dopple measurement of renal interlobar vein impedance index in uncomplicated and preeclamptic pregnancies. Hypertens Pregnancy 28: 23-33.

15. Gyselaers W, Molenberghs G, Mesens T, Peeters L (2009) Maternal hepatic vein Doppler velocimetry during uncomplicated pregnancy and pre-eclampsia. Ultrasound Med Biol 35: 1278-1283.

16. Turan OM, Turan S, Gungor S, Berg C, Moyano D, et al. (2008) Progression of Doppler abnormalities in intrauterine growth restriction. Ultrasound Obstet Gynecol 32: 160-167.

17. Bamfo JE, Kametas NA Chambers JB, Nicolaides KH (2007) Maternal cardiac function in fetal growth-restricted and non-growth-restricted small-forgestational age pregnancies. Ultrasound Obstet Gynecol 29: 51-57.

18. Tomsin K, Mesens T, Molenberghs G, Gyselaers W (2011) Diurnal and positioninduced variability of impedance cardiography measurements in healthy subjects. Clin Physiol Funct Imaging 31: 145-150.

19. Benedetto C, Marozio L, Giarola M, Chiarolini L, Maulà V, et al. (1998) Twentyfour hour blood pressure monitoring in early pregnancy: Is it predictive of pregnancy-induced hypertension and preeclampsia? Acta Obstet Gynecol Scand 77: 14-21.

20. Vonck S, Staelens AS, Bollen I (2016) Why non-invasive materna hemodynamics assessment is clinically relevant in early pregnancy: A literature review. BMC Pregnancy Childbirth 16: 302.

21. Hermida RC, Ayala DE (2001) Evaluation of the blood pressure load in the diagnosis of hypertension in pregnancy. Hypertension 38: 723-729.

22. Bujold E, Roberge S, Lacasse Y, Bureau M, Audibert F, et al. (2010) Prevention of preeclampsia and intrauterine growth restriction with aspirin started in early pregnancy: A meta-analysis. Obstet Gynecol 116: 402-414. 
Citation: Vonck S, Oben J, Staelens AS, Lanssens D, Molenberghs G, et al. (2017) Optimization of Simple Sphygmomanometric Blood Pressure Measurement in Routine Prenatal Care. Health Care Current Reviews 5: 185. doi: 10.4172/2375-4273.1000185

23. Roberge S, Villa $P$, Nicolaides K, Giguère $Y$, Vainio $M$, et al. (2012) Early administration of low-dose aspirin for the prevention of preterm and term preeclampsia: A systematic review and meta-analysis. Fetal Diagn Ther 31 : $141-146$

24. Hofmeyr GJ, Lawrie TA, Atallah AN, Duley L (2010) Calcium supplementation during pregnancy for preventing hypertensive disorders and related problems. Cochrane Database Syst Rev CD001059.

25. Aberg A (1992) Diagnostic methods for pregnancy hypertension. Significance of standardized conditions. Int J Technol Assess Health Care 8: 72-74.

26. Ayala DE, Hermida RC (2013) Ambulatory blood pressure monitoring for the early identification of hypertension in pregnancy. Chronobiology International 30: 233-259.

27. Penny JA, Halligan AW, Shennan AH, Lambert PC, Jones DR, et al. (1998) Automated, ambulatory, or conventional blood pressure measurement in pregnancy: Which is the better predictor of severe hypertension? Am J Obstet Gynecol 178: 521-526.

28. Krzesiński P, Stańczyk A, Gielerak G, Piotrowicz K, Banak M, et al. (2016) The diagnostic value of supine blood pressure in hypertension. Arch Med Sci 12 310-318.

29. Aoki K, Sato K (1986) Decrease in blood pressure and increase in tota peripheral vascular resistance in supine resting subjects with normotension or essential hypertension. Jpn Heart J 27: 467-474

30. Lu LC, Wei TM, Li S, Ye XL, Zeng CL, et al. (2008) Differences in blood pressure readings between supine and sitting positions in hypertensive patients. Acta cardiologica 63: 707-711.

31. Mizuno H, Yanagisawa A, Shigeyama T, Taya M, Sasaki A, et al. (1997) Continuous ambulatory radionuclide monitoring of left ventricular function: effect of body position during ergometer exercise. J of nuclear medicine 38: 1669-1672.

32. Vrachatis D, Papaioannou TG, Konstantopoulou A, Nasothimiou EG Millasseau S, et al. (2014) Effect of supine versus sitting position on noninvasive assessment of aortic pressure waveform: A randomized cross-over study. J Hum Hypertens 28: 236-241.
33. Ayala DE, Hermida RC, Mojon A, Fernandez JR, Iglesias M (1997) Circadian blood pressure variability in healthy and complicated pregnancies. Hypertension 30: $603-610$

34. Peek M, Shennan A, Halligan A, Lambert PC, Taylor DJ, et al. (1996) Hypertension in pregnancy: which method of blood pressure measurement is most predictive of outcome? Obstet Gynecol 88: 1030-1033.

35. Benedetto C, Zonca M, Marozio L, Dolci C, Carandente F, et al. (1996) Blood pressure patterns in normal pregnancy and in pregnancy-induced hypertension, preeclampsia and chronic hypertension. Obstet Gynecol 88: 503-510.

36. Henriksen T (1998) Measurement of blood pressure in pregnancy. Tidsskr Nor Laegeforen 118: 735-737.

37. Burgess SE, MacLaughlin EJ, Smith PA, Salcido A, Benton TJ (2011) Blood pressure rising: differences between current clinical and recommended measurement techniques. J Am Soc Hypertens 5: 484-488.

38. Pickering TG, Hall JE, Appel LJ, Falkner BE, Graves J, et al. (2005) Recommendations for blood pressure measurement in humans and experimental animals: Part 1: blood pressure measurement in humans: A statement for professionals from the Subcommittee of Professional and Public Education of the American Heart Association Council on High Blood Pressure Research. Hypertension 45: 142-161.

39. Brown MA, Simpson JM (1992) Diversity of blood pressure recording during pregnancy: implications for the hypertensive disorders. Med J Aust 156: 306308.

40. Perry IJ, Wilkinson LS, Shinton RA, Beevers DG (1991) Conflicting views on the measurement of blood pressure in pregnancy. $\mathrm{Br} \mathrm{J}$ Obstet Gynaecol 98 : 241-243.

41. Ciccone MM, Aquilino A, Cortese F, Scicchitano P, Sassara M, et al. (2010) Feasibility and effectiveness of a disease and care management model in the primary health care system for patients with heart failure and diabetes (Project Leonardo). Vasc Health Risk Manag 6: 297-305. 